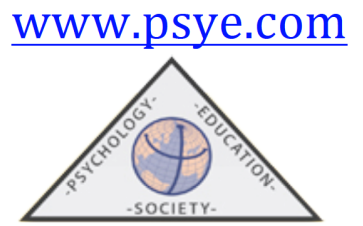

\title{
Presencia de las TIC en el diseño de un instrumento BARS para la valoración de la eficiencia del profesorado en modalidades de enseñanza online
}

\author{
Luis MATOSAS-LÓPEZ ${ }^{1}$, Cesar BERNAL-BRAVO ${ }^{1}$
}

\author{
${ }^{1}$ Universidad Rey Juan Carlos
}

(Recibido el 30 de Mayo de 2019, Aceptado el 18 de Septiembre de 2019)

\begin{abstract}
RESUMEN: Pocos son los estudios que analizan la presencia de las Tecnologías de la Información y la Comunicación (TIC) en los sistemas de valoración de la calidad docente. El presente trabajo revela el peso de las TIC en las etapas del diseño de un instrumento con episodios de comportamiento, destinado a evaluar la eficiencia del profesorado en modalidades online.

La investigación, siguiendo la metodología Behavioral Anchored Rating Scales (BARS), implica en la construcción del mencionado instrumento a un total de 477 estudiantes y seis profesores de grados online de la Universidad Rey Juan Carlos.

Los resultados revelan una elevada presencia de episodios de comportamiento con elementos TIC, tanto en las diferentes etapas del proceso de construcción como en el propio instrumento final. En este último, el $94.00 \%$ de los episodios de comportamiento recogen algún tipo de alusión a las TIC. Los hallazgos revelan que el Learning Management System (LMS), las video clases y la videoconferencia con $63.83 \%, 34.04 \%$ y $29.79 \%$ respectivamente - son los recursos tecnológicos con mayor presencia en los episodios recogidos en el instrumento de medición resultante. Asimismo, los resultados muestran el peso específico de las TIC en las siguientes categorías de la docencia online: introducción de la asignatura, resolución de dudas, capacidad explicativa y facilidad de seguimiento.
\end{abstract}

Palabras clave: educación superior; eficiencia docente; TIC; episodios de comportamiento; modalidades online

\section{Presence of ICT in the design of BARS for the assessment of teaching efficiency in online modalities}

\begin{abstract}
Few studies analyze the presence of Information and Communication Technologies (ICT) in teaching quality assessment systems. This work reveals the importance of ICT in the stages of the design process of an assessment instrument with behavioral episodes, aimed at evaluating teaching efficiency in online modalities.

The research, following the Behavioral Anchored Rating Scales (BARS) methodology, involves in the construction of the assessment instrument 477 students and six professors of online programs at Rey Juan Carlos University.

The results reveal a high presence of behavioral episodes with ICT elements, both in the different stages of the construction process and in the final instrument. In the resulting assessment instrument $94.00 \%$ of the behavioral episodes include mentions to ICT. The findings reveal that Learning
\end{abstract}


Management System (LMS), video lectures and videoconferencing - with 63.83\%, 34.04\% and 29.79\% respectively - are the technological resources with the greatest presence in the episodes included in the resulting assessment instrument. Likewise, the results show the weight of ICT in the following categories of online teaching: course introduction, dealing with doubts, explanatory capacity and follow-up easiness.

Key words: higher education; teaching efficiency; ICT; behavioral episodes; online modalities

Correspondencia: Luis Matosas-López, Universidad Rey Juan Carlos, Calle Tulipán, s/n, 28933 Móstoles, Madrid. E-mail: luis.matosas@urjc.es

\section{Introducción}

Las Tecnologías de la Información y la Comunicación (TIC) se han convertido en un agente de cambio social que ha llegado a integrarse en todos los ámbitos de nuestra vida (Domingo-Coscollola \& Marquès-Graells, 2011; Ureña López, 2016). Esta plena integración en la sociedad no ha dejado de lado la esfera educativa. García-Peñalvo y Seoane-Pardo (2015) afirman que la incursión de las TIC en el ámbito de la enseñanza ha supuesto un punto de inflexión en la forma de abordar los procesos formativos y este cambio se ha visto, sin lugar a dudas, plasmado tanto en las fases más tempranas del sistema educativo como en la etapa universitaria.

Dentro de las etapas de educación infantil, primaria y secundaria son muchos los estudios que exploran el fenómeno de la integración de las TIC desde diferentes perspectivas (Area Moreira, Hernández Rivero, \& Sosa Alonso, 2016; Llamas Salguero \& Macías Gómez, 2018; López Gil \& Bernal Bravo, 2016). En estas etapas, sobresalen sobre el resto las investigaciones en torno a las metodologías de aprendizaje colaborativas con TIC (GarcíaValcárcel, Basilotta-Gómez, \& López-García, 2014; García-Valcárcel \& Tejedor Tejedor, 2018), los estudios sobre el uso de pizarras digitales interactivas (Coscollola, 2016), o los trabajos sobre el empleo de videojuegos educativos (Evaristo Chiyong, Navarro Fernández, Vega Velarde, \& Nakano Osores, 2016), entre otros.

En el contexto universitario, las posibilidades de implementación de las TIC abren si cabe un abanico de opciones aún más amplio. Así, son numerosas las investigaciones que han tratado el tema del empleo de las TIC dentro de colectivos universitarios e instituciones de educación superior (Alba Pastor, 2005; Ferreiro Gravie, 2012; García-Valcárcel \& TejedorTejedor, 2009; García-Vera, Roig-Vila, \& García, 2016; Gómez Ortega, Jiménez García, \& Gumbau Mezquita, 2017; Vázquez-Cano, Meneses, \& García-Garzón, 2017). La revisión de diversos estudios realizada por Guerra, González y García (2010) concluye que la aplicación de las TIC en la universidad gira en torno a varios ejes. Entre estos destacan: las posibilidades didácticas para la innovación; la necesidad de formación del profesorado; su integración en el día a día de la vida académica; la contextualización de su enseñanza en situaciones concretas; o los planes estratégicos de implantación institucional. Dentro del ámbito de la educación superior cabe resaltar, también, los estudios realizados sobre: la aplicación de Personal Learning Environments (PLE) en el desarrollo de entornos de instrucción adaptados (López Vicent, González Calatayud, Aguiar Perera, \& Artiles Rodríguez, 2017); el uso de Massive Open Online Courses (MOOC) como extensión de los procesos de formación (Gómez Galán, 
2014); el empleo de tabletas y smartphones con distintas funcionalidades (Ruiz de Miguel, 2016); o la implementación de laboratorios virtuales para la realización de ensayos de manera remota (Potkonjak et al., 2016).

Sin embargo, aunque la integración de las TIC en el contexto universitario ha sido objeto de extensas investigaciones, pocos son los estudios dedicados a explorar la representación de la tecnología en los sistemas de valoración del profesorado. Esta situación se debe, en parte, a que los sistemas de evaluación docente empleados en la mayoría de las instituciones emplean instrumentos de medición con escalas Likert. En estos cuestionarios, el alumno se limita a manifestar en una escala ordinal su nivel de acuerdo (generalmente en un grado de 1 a 5) con uno o varios ítems descriptivos de la categoría docente a evaluar.

Este tipo de cuestionarios, a pesar de estar ampliamente aceptados como instrumentos fiables y válidos para evaluar la calidad docente (Lizasoain-Hernández, Etxeberria-Murgiondo \& Lukas-Mujika, 2017; Zhao \& Gallant, 2012), presentan también importantes limitaciones para reflejar de forma nítida muchos de los matices propios de la labor del profesorado. Así, por ejemplo, Spooren, Mortelmans y Denekens (2007) señalan que cuando estos instrumentos de medición emplean un único ítem para evaluar la categoría docente objeto de estudio, las escalas Likert plantean dificultades para interpretar con claridad las respuestas obtenidas en la evaluación. Asimismo, Richardson (2005) apunta las carencias de este tipo de cuestionarios a la hora de reflejar las innovaciones curriculares acontecidas en el ámbito de la educación superior.

Los problemas señalados de falta de claridad en la interpretación de las respuestas e incapacidad para reflejar las innovaciones experimentadas en la enseñanza hacen que los instrumentos con escalas Likert resulten insuficientes para mostrar la incidencia efectiva del uso de las TIC en la valoración del profesorado. Estas limitaciones ocasionan que los aspectos relativos al uso que el docente hace de la tecnología en su desempeño se diluyan a lo largo del proceso de evaluación.

Los cuestionarios Likert dificultan que el estudiante pueda dar su visión sobre qué recursos tecnológicos son más adecuados para cada categoría de la docencia e impiden, en consecuencia, que el profesor obtenga la retroalimentación que necesita para conocer la manera de aprovechar esos recursos. Esta situación dificulta la aplicación del fin formativo de la evaluación y obstaculiza la adopción de medidas por parte del docente de cara a la mejora de la calidad del curso. Si consideramos la influencia que, hoy por hoy, tiene el componente tecnológico en las metodologías docentes, esta incapacidad de los cuestionarios Likert para plasmar la incidencia real del uso de las TIC en las distintas vertientes de la docencia resulta especialmente crítica.

Sin embargo, aunque las encuestas de satisfacción del alumnado aplicadas mediante cuestionarios Likert son el sistema de evaluación del profesorado más extendido, también existen otras alternativas. Diferentes autores afirman, por ejemplo, que los sistemas de valoración docente pueden ser mejorados empleando episodios de comportamiento en lugar de elementos numéricos como puntos de anclaje en el instrumento de medición (Martin-Raugh, Tannenbaum, Tocci, \& Reese, 2016; Matosas-López, Leguey-Galán, \& Doncel-Pedrera, 2019). Los instrumentos con episodios de comportamiento, Behavioral Anchored Rating Scales o BARS, permiten recoger múltiples matices del desempeño del profesor para cada una de las 
categorías de la docencia. Por consiguiente, en opinión de los autores, la utilización de BARS permite reflejar de manera clara e inequívoca el peso de las TIC en la labor del docente.

Objetivo

El objetivo del presente trabajo es mostrar la incidencia que tienen las TIC en los episodios de comportamiento considerados en las sucesivas etapas del diseño de un instrumento de medición BARS destinado a evaluar la eficiencia del profesorado de modalidades de enseñanza online.

\section{Metodología}

\section{Método BARS}

La construcción del instrumento de medición BARS adopta como referencia la metodología originalmente descrita por Smith y Kendall (1963). Esta metodología toma las categorías a considerar en la actividad analizada, recopilando para cada una de ellas episodios de comportamientos eficientes e ineficientes. Episodios que, tras sucesivas fases de cribado y categorización, sirven para representar los puntos de anclaje en la escala final. Estos episodios de comportamiento reemplazan así a los elementos numéricos empleados en los instrumentos de valoración docente con escalas Likert.

Utilizando como referencia el método previamente señalado, se aborda la construcción del mencionado instrumento de valoración del profesorado empleando el método BARS con agrupación de aspectos básicos de comportamiento (ACB) postulado por Matosas-López, Aguado-Franco y Gómez-Galán (2019). Este método, en línea con otras investigaciones sobre diseño de BARS (Kell et al., 2017; Klieger et al., 2018), presenta un proceso de construcción con siete etapas diferenciadas:

- Etapa 1. Descripción de las categorías de la docencia en modalidades online

- Etapa 2. Recopilación de episodios de comportamiento del docente en modalidades online

- Etapa 3. Depurado de episodios de comportamiento

- Etapa 4. Reubicación de episodios de comportamiento en las categorías de la docencia

- Etapa 5. Agrupación de episodios de comportamiento en subgrupos de ACB

- Etapa 6. Evaluación dual de episodios de comportamiento

- Etapa 7. Selección de ACB y generación de la escala final

En primer lugar, un grupo de profesores realiza una breve descripción de las diez categorías objeto de estudio. Nueve de estas categorías reflejan las diferentes vertientes a considerar en la evaluación de la calidad docente en esta modalidad de enseñanza. Estas categorías son: introducción a la asignatura, descripción del sistema de evaluación, gestión del tiempo, coherencia organizativa, implementación del sistema de evaluación, resolución de dudas, capacidad explicativa, disponibilidad general y facilidad de seguimiento. La décima categoría, satisfacción general, representa la satisfacción global del estudiante con el desempeño del profesor de modalidad online.

En la segunda etapa, un colectivo de estudiantes organizados en sucesivas entrevistas personales y grupales proporciona episodios de comportamientos (eficientes e ineficientes) en (c) Psy, Soc, \& Educ, 2020, Vol. 12(1) 
la labor docente en entornos virtuales. Un total de 387 episodios de comportamiento son recopilados.

Durante la tercera etapa, el grupo de profesores revisa todos los episodios de comportamiento recogidos durante la etapa anterior y procede a eliminar aquellos redundantes o ambiguos. Tras esta etapa, el número de episodios de comportamiento queda reducido a 303.

En la etapa cuarta, otro colectivo de alumnos reubica los episodios de comportamiento anteriores en las diez categorías objeto de estudio. Aquellos episodios de comportamiento que no son reasignados en la categoría para la cual fueron propuestos por al menos un $80 \%$ de los estudiantes son eliminados del proceso. En este paso, 282 episodios de comportamiento resultan reubicados satisfactoriamente.

Durante la quinta etapa, el grupo de profesores interviene de nuevo para revisar los episodios de comportamiento restantes. Aquí, en línea con Matosas (2018), los docentes proceden a reagrupar los episodios de cada categoría en subcategorías con aspectos comunes que pasan a denominarse ACB. Los profesores detectan que dentro de cada categoría los episodios de comportamiento pueden ser reagrupados invariablemente en cuatro ACB claramente diferenciados. En este paso, los episodios de comportamiento quedan sintetizados en 40 subcategorías de ACB.

En la etapa sexta, los cuartetos de ACB detectados en cada categoría se mezclan entre sí para generar todas las combinaciones posibles. Posteriormente, un tercer colectivo de estudiantes procede a valorar numéricamente las combinaciones de ACB generadas. Con estas valoraciones se calculan los promedios y las desviaciones típicas de cada combinación dentro de su categoría. El valor del promedio identifica el lugar que esa combinación de ACB podrá ocupar en la escala final; mientras que la desviación típica representa el grado de acuerdo entre los alumnos al colocar esa combinación en el lugar indicado por el valor promedio.

Finalmente, en la etapa séptima, los investigadores conforman la escala final seleccionando las combinaciones de ACB que presentan valores de desviación típica más bajos. El instrumento resultante consta de diez categorías con cinco puntos de anclaje en cada una de ellas; estando cada punto de anclaje, a su vez, representado por una combinación de ACB. En consecuencia, el número de ejemplos conductuales reflejado en el instrumento final asciende a $50 \mathrm{ACB}$, cinco por categoría.

\section{Participantes}

La investigación toma como objeto de estudio los diez grados de modalidad online de la Universidad Rey Juan Carlos (en adelante URJC). Los grados considerados son: Grado en Administración y Dirección de Empresas, Grado en Publicidad y Relaciones Públicas, Grado en Contabilidad y Finanzas, Grado en Derecho, Grado en Periodismo, Grado en Ciencia, Grado en Gestión e Ingeniería de Servicios, Grado en Educación Infantil, Grado en Educación Primaria y Grado en Turismo. La muestra está constituida por 477 estudiantes de los mencionados grados y por un panel de seis profesores, de la propia universidad, con experiencia docente en entornos virtuales. En línea con otros trabajos en el contexto de la educación superior (Gámiz Sánchez \& Gallego Arrufat, 2015; Pérez-Escoda, García-Ruiz, \& Aguaded-Gómez, 2018), los participantes, tanto alumnos como docentes, son seleccionados por 
muestreo no probabilístico incidental o de conveniencia. La edad promedio de los estudiantes se sitúa en 27.12 años $(D T=4.24)$, siendo la distribución de género de $57.24 \%$ (femenino), $42.76 \%$ (masculino). La edad promedio de los profesores, por su parte, es de $42.31(D T=1.91)$ contándose con idéntico número de hombres y mujeres.

\section{Procedimiento}

El presente estudio, profundizando en el trabajo desarrollado por Matosas-López, Aguado-Franco y Gómez-Galán (2019) sobre el proceso de construcción de un instrumento BARS para la evaluación del profesorado de enseñanzas virtuales, hace una exploración descriptiva de la incidencia de las TIC en las distintas etapas del diseño del cuestionario. Para ello, los investigadores examinan las frecuencias de aquellos episodios de comportamiento que recogen alusiones a elementos TIC durante las etapas dos, tres, cuatro, cinco y siete del proceso de construcción. Las etapas uno y seis no son consideradas a efectos de exploración de frecuencias. La etapa primera, por limitarse a la mera descripción de las categorías y no recoger ejemplos concretos sobre la labor del docente. La etapa sexta, por su parte, por no presentar variación alguna con respecto a la etapa quinta, en lo que a ejemplos de comportamiento se refiere.

Los elementos TIC fiscalizados durante el proceso son los siguientes: Learning Management System (LMS), video clases, videoconferencia, foros, email, chat, presentaciones interactivas Prezi ${ }^{\circledR}$ o Sway ${ }^{\circledR}$, podcast, test auto evaluables y video presentación.

\section{Resultados}

Los resultados expuestos en la tabla 1 muestran la presencia de episodios de comportamiento con alusiones a las TIC en las cinco fases del proceso de construcción analizadas. La presencia de episodios con elementos TIC oscila entre el $62.50 \%$ reflejado en la etapa de agrupación de episodios de comportamiento en subgrupos de ACB (etapa 5) y el $94.00 \%$ mostrado en la generación del instrumento final (etapa 7).

Tabla 1. Episodios de comportamiento con alusiones a las TIC por etapa del proceso de construcción

\begin{tabular}{|c|c|c|c|}
\hline Etapa del proceso de construcción & $\begin{array}{c}\mathrm{N}^{\circ}{ }^{\circ} \text { total de } \\
\text { episodios de } \\
\text { comportamiento }\end{array}$ & $\begin{array}{c}\mathrm{N}^{\circ}{ }^{0} \text { de episodios } \\
\text { de } \\
\text { comportamiento } \\
\text { con alusiones } \\
\text { TIC }\end{array}$ & $\begin{array}{c}\% \text { de episodios } \\
\text { de } \\
\text { comportamiento } \\
\text { con alusiones } \\
\text { TIC }\end{array}$ \\
\hline $\begin{array}{l}\text { Etapa 2. Recopilación de episodios de } \\
\text { comportamiento del docente en modalidades online }\end{array}$ & 387 & 289 & $74.68 \%$ \\
\hline Etapa 3. Depurado de episodios de comportamiento & 303 & 275 & $90.76 \%$ \\
\hline $\begin{array}{l}\text { Etapa 4. Reubicación de episodios de } \\
\text { comportamiento en las categorías de la docencia }\end{array}$ & 282 & 197 & $69.86 \%$ \\
\hline $\begin{array}{l}\text { Etapa 5. Agrupación de episodios de } \\
\text { comportamiento en subgrupos de ACB }\end{array}$ & 40 & 25 & $62.50 \%$ \\
\hline $\begin{array}{l}\text { Etapa 7. Selección de ACB y generación de la escala } \\
\text { final }\end{array}$ & 50 & 47 & $94.00 \%$ \\
\hline
\end{tabular}


Fuente: Elaboración propia

Por otro lado, el desglose de las frecuencias de episodios de comportamiento por categoría y etapa del proceso de construcción revela una elevada presencia de alusiones a elementos TIC en las diez categorías examinadas (ver Tabla 2).

Tabla 2. Episodios de comportamiento con alusiones a las TIC por etapa del proceso de construcción y categoría

\begin{tabular}{|c|c|c|c|c|c|}
\hline Categoría & $\begin{array}{c}\text { Etapa 2 } \\
\text { Recopilación de } \\
\text { episodios de } \\
\text { comportamiento } \\
\text { del docente en } \\
\text { modalidades } \\
\text { online } \\
\end{array}$ & $\begin{array}{c}\text { Etapa } 3 \\
\text { Depurado de } \\
\text { episodios de } \\
\text { comportamiento }\end{array}$ & $\begin{array}{c}\text { Etapa } 4 \\
\text { Reubicación de } \\
\text { episodios de } \\
\text { comportamiento } \\
\text { en las categorías } \\
\text { de la docencia }\end{array}$ & $\begin{array}{c}\text { Etapa 5 } \\
\text { Agrupación de } \\
\text { episodios de } \\
\text { comportamiento } \\
\text { en subgrupos de } \\
\text { ACB }\end{array}$ & $\begin{array}{c}\text { Etapa } 7 \\
\text { Selección final } \\
\text { de ACB y } \\
\text { generación de } \\
\text { la escala } \\
\end{array}$ \\
\hline $\begin{array}{l}\text { Introducción a la } \\
\text { asignatura }\end{array}$ & 47 & 46 & 35 & 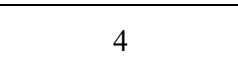 & 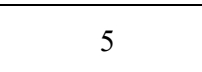 \\
\hline $\begin{array}{l}\text { Descripción del sistema de } \\
\text { evaluación }\end{array}$ & 27 & 26 & 15 & 3 & 5 \\
\hline Gestión del tiempo & 29 & 28 & 19 & 3 & 5 \\
\hline Disponibilidad general & 21 & 21 & 15 & 2 & 5 \\
\hline Coherencia organizativa & 23 & 22 & 15 & 2 & 5 \\
\hline $\begin{array}{l}\text { Implementación del } \\
\text { sistema de evaluación }\end{array}$ & 10 & 10 & 5 & 1 & 2 \\
\hline Resolución de dudas & 35 & 32 & 25 & 3 & 5 \\
\hline Capacidad explicativa & 34 & 32 & 27 & 2 & 5 \\
\hline Facilidad de seguimiento & 35 & 31 & 27 & 3 & 5 \\
\hline Satisfacción general & 28 & 27 & 14 & 2 & 5 \\
\hline Total & 289 & 275 & 197 & 25 & 47 \\
\hline
\end{tabular}

Fuente: Elaboración propia

En las etapas dos, tres, y cuatro las categorías "introducción de la asignatura", "resolución de dudas", "capacidad explicativa" y "facilidad de seguimiento" son las que presentan un mayor número de alusiones a las TIC. La categoría "introducción de la asignatura" aglutina episodios de comportamiento en los que se alude: a la realización del video de presentación del docente o al uso del LMS del curso para cargar el calendario de actividades, la guía docente y la guía de estudio de la asignatura. En la categoría "resolución de dudas" se mencionan las funcionalidades de videoconferencia, foro, email o chat para abordar la comunicación entre estudiante y profesor durante el curso. La categoría "capacidad explicativa" recoge, a su vez, alusiones a las video clases y otros recursos educativos de carácter digital como las presentaciones interactivas o los podcasts. Por su parte, la categoría "facilidad de seguimiento" contiene referencias al uso de los foros de la asignatura y las video clases tanto en su vertiente sincrónica como asincrónica.

En la etapa cinco, la categoría "introducción a la asignatura" es la que muestra un mayor número de menciones a elementos TIC. Asimismo, en la etapa siete, la cual se corresponde con el instrumento de medición final, se observa similar número de alusiones a elementos TIC en 
nueve de las diez categorías. La excepción es la categoría relativa a la implementación del sistema de evaluación. En esta última, se menciona el uso de las herramientas de comunicación del LMS para abordar las tareas de revisión en las actividades evaluables del curso.

La tabla 3, por su parte, expone la presencia de cada componente tecnológico en el instrumento de medición final (etapa 7). El LMS del curso, con un $63.83 \%$ de las menciones, es el recurso con mayor presencia. En segundo lugar, presente en un $34.04 \%$ de los episodios de comportamiento, encontramos las video clases. Tras las video clases, las herramientas TIC que parecen tener más peso en el instrumento de medición son aquellas empleadas para regular los flujos de comunicación entre estudiante y profesor. Aquí encontramos: la videoconferencia, los foros, el email y la funcionalidad de chat.

Tabla 3. Presencia de episodios de comportamiento con alusiones a las TIC en el instrumento final

\begin{tabular}{|c|c|c|}
\hline Elemento TIC & $\begin{array}{l}\mathrm{N} .^{\circ} \text { de episodios de } \\
\text { comportamiento con } \\
\text { alusiones TIC }\end{array}$ & $\begin{array}{c}\% \text { de episodios de } \\
\text { comportamiento con alusiones } \\
\text { TIC sobre el total de episodios } \\
\text { del instrumento final* }\end{array}$ \\
\hline LMS & 30 & $63.83 \%$ \\
\hline Video clases & 16 & $34.04 \%$ \\
\hline Videoconferencia & 14 & $29.79 \%$ \\
\hline Foros & 10 & $21.28 \%$ \\
\hline Email & 10 & $21.28 \%$ \\
\hline Chat & 10 & $21.28 \%$ \\
\hline Presentaciones interactivas $\left(\right.$ Prezi $^{\circledR}$ o Sway $\left.{ }^{\circledR}\right)$ & 4 & $8.51 \%$ \\
\hline Podcast & 4 & $8.51 \%$ \\
\hline Test auto evaluables & 3 & $6.38 \%$ \\
\hline Video presentación & 2 & $4.26 \%$ \\
\hline
\end{tabular}

Fuente: Elaboración propia

* Un mismo episodio de comportamiento puede contener alusiones a varios elementos TIC

Con una menor incidencia, observamos también la presencia de recursos educativos digitales como las presentaciones interactivas y los podcasts, ambos presentes en el $8.51 \%$ de los episodios de comportamiento. Por último, los elementos TIC con una menor presencia en el instrumento de medición generado son los test auto evaluables y el video en su papel de herramienta de presentación de la asignatura. Estos presentan porcentajes del 6.38\% y 4.26\% respectivamente.

El LMS, como recurso tecnológico con mayor presencia en el instrumento resultante, goza de especial relevancia en la categoría "satisfacción general". La figura 1 muestra la escala generada en el instrumento final (etapa 7) para esta categoría. Esta escala refleja cómo la satisfacción global del alumno con el desempeño del docente contempla dos aspectos diferenciados en lo que atañe al uso del LMS. Por un lado, el alumno otorga importancia al manejo que el profesor hace de los recursos propios del LMS, por otro al diseño y estructuración de la asignatura dentro de la plataforma. 
Figura 1. Presencia de episodios de comportamiento con alusiones a las TIC en la escala de la categoría "satisfacción general" en el instrumento final

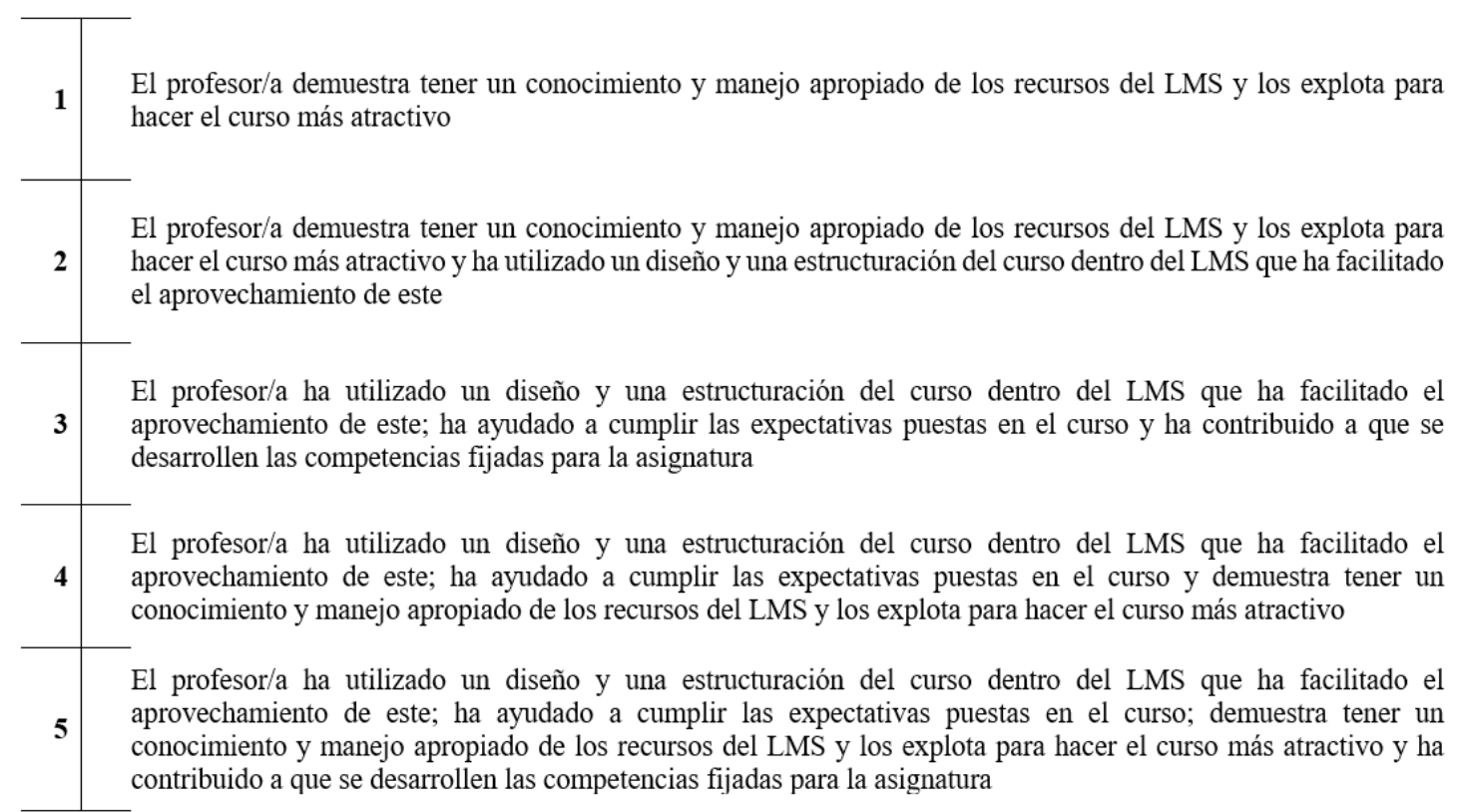

Fuente: Elaboración propia

La explotación que el profesor hace del LMS y los recursos de este para mejorar el atractivo del curso aparecen en los episodios de comportamiento primero, segundo, cuarto y quinto de la escala final. Por su parte, el diseño y estructuración del curso en la plataforma queda reflejado en los episodios segundo, tercero, cuarto y quinto para la categoría indicada. Observamos, por tanto, cómo los dos primeros puntos de anclaje de la escala de esta categoría quedan constituidos íntegramente por los episodios de comportamiento que aluden al uso que el docente hace del LMS del curso.

\section{Conclusiones}

El análisis de las fases del diseño de un instrumento de valoración docente para modalidades online con episodios de comportamiento refleja de manera clara e inequívoca la incidencia que las TIC tienen en los sistemas de valoración del profesorado en entornos virtuales. En línea con García-Peñalvo y Pardo (2015), los resultados expuestos subrayan la relación entre el uso de la tecnología y la calidad de la docencia en modalidades de enseñanza online en el ámbito universitario.

La elevada presencia de elementos tecnológicos a lo largo de las cinco etapas examinadas en el proceso de construcción apunta un alto grado de acuerdo por parte del alumnado a la hora de: a) entender la funcionalidad que las TIC brindan en cada una de las categorías de la docencia online, y b) vincular la explotación de cada recurso TIC a un determinado nivel de desempeño en la labor docente. Lo primero queda patente en las etapas dos, tres, cuatro y cinco; etapas en las que se formulan, depuran, ordenan y agrupan los episodios de comportamiento en las distintas categorías. Lo segundo se pone de manifiesto en 
la etapa séptima, etapa en la que se observa cómo las combinaciones de ACB han sido relacionadas, por el estudiante, con los distintos niveles de eficiencia docente en la escala final.

Las elevadas frecuencias de episodios de comportamiento con elementos TIC en las diez categorías consideradas muestra, en opinión de los autores, la importancia que el componente tecnológico tiene a la hora de evaluar la calidad en cualquiera de las vertientes de la docencia en modalidades online. Asimismo, los hallazgos realizados revelan el especial peso de las TIC en categorías de la docencia como: introducción de la asignatura, resolución de dudas, capacidad explicativa y facilidad de seguimiento.

La observación de los episodios de comportamiento que sirven para constituir el instrumento final confirma, una vez más, el interés que las TIC tienen en la valoración de la docencia online. Consistentes con el estudio realizado por Horvat, Dobrota, Krsmanovic y Cudanov (2015) sobre la percepción de los LMS en entornos virtuales, los resultados muestran la importancia de una adecuada explotación de la plataforma en estas modalidades. De igual forma, en línea con otros autores (Soffer, Kahan, \& Livne, 2017), los hallazgos constatan el peso de las video clases como recurso determinante en la eficiencia de la enseñanza en línea. Las video clases, no solo destacan por su atractivo como herramienta de aprendizaje sincrónico, sino que también tienen un valor como recurso asincrónico. Lo primero cuando se emplean para exponer contenidos en directo, lo segundo cuando son grabadas para su posterior visualización en diferido, en aquellos casos en los que el alumno no puede estar conectado en el momento de la emisión.

En consonancia con Sebastianelli y Tamimi (2011), los resultados revelan también la importancia que tienen las herramientas TIC orientadas a la comunicación (videoconferencia, foros, email, chat) a la hora de evaluar el desempeño del profesor en la enseñanza online. Estos hallazgos, en línea con Rovai et al. (2006), evidencian la dificultad inherente a la gestión de la comunicación estudiante-profesor en los cursos desarrollados en entornos virtuales. Aunque son muchos los recursos de comunicación que las TIC ponen al servicio de estas modalidades, tanto de manera diferida (email o foros) como de manera sincrónica (videoconferencia o chat), el carácter impersonal y alejado de las interacciones estudiante-profesor sigue siendo un gran reto para las instituciones educativas de todo el mundo.

Finalmente, el estudio revela, también, la incidencia de las TIC en la satisfacción del alumno con la labor del docente. La escala representativa de la categoría satisfacción general en el instrumento final muestra cómo el nivel básico de eficiencia esperado por el estudiante de modalidad online queda supeditado al aprovechamiento que el profesor hace del LMS del curso. En este aprovechamiento del LMS se contempla tanto el uso que el profesor hace de los recursos de este como el diseño y estructuración de la asignatura en la plataforma. Este último hecho está alineado con el trabajo de Palloff y Pratt (2001), estudio que destaca cómo la estructuración del curso en el LMS resulta clave a la hora de evaluar la calidad docente en modalidades de enseñanza a distancia. Este hallazgo corrobora el potencial del LMS como elemento vertebrador del curso, y no solo como lugar de integración de contenidos y comunicación entre estudiante y docente.

Los episodios de comportamiento recogidos durante el presente estudio exhiben alusiones recurrentes al empleo de múltiples recursos TIC con diferentes propósitos y finalidades. Los estudiantes de estas modalidades demandan que el profesor, además de tener 
un dominio del componente tecnológico, realice también una aplicación de este en todas las vertientes de la docencia. Los resultados apuntan, por tanto, que el empleo de las TIC está plenamente asimilado por el estudiante de modalidades en línea, y que además su uso confiere a la propia modalidad de enseñanza su identidad digital.

Para terminar, los autores concluyen que la utilización de escalas con episodios de comportamiento en la valoración del profesorado en entornos online permite al instrumento de medición mostrar la incidencia y el impacto real de las TIC en la labor docente en estas modalidades de enseñanza.

\section{Referencias}

Alba Pastor, C. (2005). El profesorado y las tecnologías de la información y la comunicación en el proceso de convergencia al espacio europeo de educación superior. Revista de educación, (337), 13-36. https://dialnet.unirioja.es/servlet/articulo?codigo=1271345

Area Moreira, M., Hernández Rivero, V. M., \& Sosa Alonso, J. J. (2016). Modelos de integración didáctica de las TIC en el aula. Comunicar, 24(47), 79-87. https://doi.org/http://dx.doi.org/10.3916/C47-2016-08

Coscollola, M. U. I. de C. D. (2016). Pizarra digital interactiva en el aula: Uso y valoraciones sobre el aprendizaje. Estudios sobre Educación, 20, 99-116. Recuperado de http://www.unav.edu/publicaciones/revistas/index.php/estudios-sobreeducacion/article/view/4475

Domingo-Coscollola, M., \& Marquès-Graells, P. (2011). Aulas 2.0 y uso de las TIC en la práctica docente. Comunicar, 19(37), 169-175. https://doi.org/10.3916/C37-2011-03-09

Evaristo Chiyong, I., Navarro Fernández, R., Vega Velarde, V., \& Nakano Osores, T. (2016). Uso de un videojuego educativo como herramienta para aprender historia del Perú. La Revista Iberoamericana de Educación a Distancia (RIED), 18(2), 35-52. Recuperado de http://revistas.uned.es/index.php/ried/article/view/15569/14274

Ferreiro Gravie, R. F. (2012). Criterios de calidad de una buena práctica para la aplicación de las TIC a nivel de institución. Revista Complutense de Educación, 23(1), 51-60. https://doi.org/10.5209/rev_RCED.2012.v23.n1.39101

Gámiz Sánchez, V., \& Gallego Arrufat, M. J. (2015). Modelo de análisis de metodologías didácticas semipresenciales en Educación Superior. Educación XX1, 19(1). https://doi.org/10.5944/educxx1.15577

García-Peñalvo, F. J., \& Seoane-Pardo, A. M. (2015). Una revisión actualizada del concepto de eLearning: Décimo aniversario. Teoría de la Educación, 16(1), 119-144. https://doi.org/10.14201/eks2015161119144

García-Valcárcel, A., Ana García-Valcárcel, \& F. J. T. (2009). Information and communication technologies in university teaching: Implications in european higher education space. International Journal of Human Sciences. Recuperado de https://www.jhumansciences.com/ojs/index.php/IJHS/article/view/762 
García-Valcárcel, A. \& Tejedor-Tejedor, F. (2009). (2014). Las TIC en el aprendizaje colaborativo en el aula de Primaria y Secundaria. Comunicar, 21(42), 65-74. https://doi.org/10.3916/C42-2014-06

García-Valcárcel, A., \& Tejedor Tejedor, F. J. (2018). Valoración del trabajo colaborativo en los procesos de enseñanza-aprendizaje en entornos escolares con alto nivel TIC. Estudios sobre Educación, 34(0), 155-175. Recuperado de https://www.unav.edu/publicaciones/revistas/index.php/estudios-sobreeducacion/article/view/8743/17439

García-Vera, V., Roig-Vila, R., \& García, P. (2016). Construcción de un instrumento para medir la utilidad percibida de las TIC usadas en la docencia por estudiantes de arquitectura técnica. Pixel-Bit. Revista de Medios y Educación, 49, 121-135.

Gómez Galán, J. (2014). El fenómeno MOOC y la universalidad de la cultura: Las nuevas fronteras de la Educación Superior. Profesorado, Revista de Currículum y Formación del Profesorado, 18(1), 73-91. Recuperado de https://recyt.fecyt.es/index.php/profesorado/article/view/41069/23349

Gómez Ortega, J., Jiménez García, T., \& Gumbau Mezquita, J. P. (2017). Universitic 2017. Análisis de las TIC en las Universidades Españolas. (J. Gómez Ortega, Ed.). Madrid: Crue Universidades Españolas.

Guerra, S., González, N., \& García, R. (2010). Utilización de las TIC por el profesorado universitario como recurso didáctico. Comunicar, 17(35), 141-148. https://doi.org/10.3916/C35-2010-03-07

Horvat, A., Dobrota, M., Krsmanovic, M., \& Cudanov, M. (2015). Student perception of Moodle learning management system: a satisfaction and significance analysis. Interactive Learning Environments, 23(4), 515-527. https://doi.org/10.1080/10494820.2013.788033

Kell, H. J., Martin-Raugh, M. P., Carney, L. M., Inglese, P. A., Chen, L., \& Feng, G. (2017). Exploring methods for developing Behaviorally Anchored Rating Scales for evaluating structured interview performance. Princeton. Recuperado de https://onlinelibrary.wiley.com/doi/full/10.1002/ets2.12152

Klieger, D. M., Kell, H. J., Rikoon, S., Burkander, K. N., Bochenek, J. L., \& Shore, J. R. (2018). Development of the Behaviorally Anchored Rating Scales for the skills demonstration and progression guide. Princeton. Recuperado de https://onlinelibrary.wiley.com/doi/full/10.1002/ets2.12210

Lizasoain-Hernández, L., Etxeberria-Murgiondo, J., \& Lukas-Mujika, J. F. (2017). Propuesta de un nuevo cuestionario de evaluación de los profesores de la Universidad del País Vasco. Estudio psicométrico, dimensional y diferencial. RELIEVE - Revista Electrónica de Investigación y Evaluación Educativa, 23(1). https://doi.org/10.7203/relieve.23.2.10436

Llamas Salguero, F., \& Macías Gómez, E. (2018). Formación inicial de docentes en educación básica para la generación de conocimiento con las tecnologías de la información y la comunicación. Revista Complutense de Educación, 29(2), 577-593. https://doi.org/10.5209/RCED.53520 
López Gil, M. M., \& Bernal Bravo, C. (2016). La cultura digital en la escuela pública. Revista Interuniversitaria de Formación del Profesorado, 85(2016), 103-110. Recuperado de https://www.redalyc.org/articulo.oa?id=27446519010

López Vicent, P., González Calatayud, V., Aguiar Perera, M. V., \& Artiles Rodríguez, J. (2017). La gestión de la información en entornos personales de aprendizaje: estudio exploratorio en alumnado de último curso de grado. Revista Complutense de Educación, 28(4), 1303-1320. https://doi.org/10.5209/RCED.51849

Martin-Raugh, M., Tannenbaum, R. J., Tocci, C. M., \& Reese, C. (2016). Behaviourally anchored rating scales: An application for evaluating teaching practice. Teaching and Teacher Education, 59, 414-419. https://doi.org/10.1016/j.tate.2016.07.026

Matosas, L. (2018). Aspectos de comportamiento básico del profesor universitario en los procesos de valoración docente para modalidades blended learning. Espacios, 39(10), 10-24. Recuperado de http://www.revistaespacios.com/a18v39n17/18391713.html

Matosas-López, L., Aguado-Franco, J. C., \& Gómez-Galán, J. (2019). Constructing an instrument with behavioral scales to assess teaching quality in blended learning modalities. Journal of New Approaches in Educational Research, 8(2), 142-165. https://doi.org/10.7821/naer.2019.7.410.

Matosas-López, L., Leguey-Galán, S. \& Doncel-Pedrera, L. M. (2019). Converting Likert scales into Behavioral Anchored Rating Scales(Bars) for the evaluation of teaching effectiveness for formative purposes. Journal of University Teaching \& Learning Practice, 16(3), 1-24. Recuperado de https://ro.uow.edu.au/jutlp/vol16/iss3/9.

Palloff, R. M., \& Pratt, K. (2001). Lessons from the cyberspace classroom: The realities of online teaching (1st Edition). San Francisco: Jossey-Bass.

Pérez-Escoda, A., García-Ruiz, R., \& Aguaded-Gómez, I. (2018). La competencia mediática en el profesorado universitario. Validación de un instrumento de evaluación. @Tic Revista D’Innovació Educativa, O(21), 1. https://doi.org/10.7203/attic.21.12550

Potkonjak, V., Gardner, M., Callaghan, V., Mattila, P., Guetl, C., Petrović, V. M., \& Jovanović, K. (2016). Virtual laboratories for education in science, technology, and engineering: A $\begin{array}{llll}\text { review. Computers } \& \text { Education, 309-327. } & \text { \& }\end{array}$ https://doi.org/10.1016/j.compedu.2016.02.002

Richardson, J. T. E. (2005). Instruments for obtaining student feedback: a review of the literature. Assessment \& Evaluation in Higher Education, 30(4), 387-415. https://doi.org/10.1080/02602930500099193

Rovai, A. P., Ponton, M. K., Derrick, M. G., \& Davis, J. M. (2006). Student evaluation of teaching in the virtual and traditional classrooms: A comparative analysis. Internet and Higher Education, 9(1), 23-25. https://doi.org/10.1016/j.iheduc.2005.11.002

Ruiz de Miguel, C. (2016). Perfil de uso del teléfono móvil e Internet en una muestra de universitarios españoles. Bordón. Revista de pedagogía, Vol. 68( $\left.\mathrm{N}^{\mathrm{o}} 3\right), 131-145$. Recuperado de https://dialnet.unirioja.es/servlet/articulo?codigo=5546265

Sebastianelli, R., \& Tamimi, N. (2011). Business Statistics and Management Science Online: Teaching Strategies and Assessment of Student Learning. Journal of Education for Business, 86(6), 317-325. https://doi.org/10.1080/08832323.2010.525545 
Smith, P. C., \& Kendall, L. M. (1963). Retranslation of expectations: An approach to the construction of unambiguous anchors for rating scales. Journal of Applied Psychology, 47(2), 149-155. https://doi.org/10.1037/h0047060

Soffer, T., Kahan, T., \& Livne, E. (2017). E-assessment of online academic courses via students' activities and perceptions. Studies in Educational Evaluation, 54, 83-93. https://doi.org/10.1016/j.stueduc.2016.10.001

Spooren, P., Mortelmans, D., \& Denekens, J. (2007). Student evaluation of teaching quality in higher education: development of an instrument based on 10 Likert-scales. Assessment \& Evaluation in Higher Education, 32(6), 667-679. https://doi.org/10.1080/02602930601117191

Ureña López, S. (2016). Dimensiones de la inclusión de las TIC en el currículo educativo: una aproximación teórica. Teoría de la Educación, 28(1), 209. https://doi.org/10.14201/teoredu2016281209223

Vázquez-Cano, E., Meneses, E. L., \& García-Garzón, E. (2017). Differences in basic digital competences between male and female university students of Social Sciences in Spain. International Journal of Educational Technology in Higher Education, 14(1). https://doi.org/10.1186/s41239-017-0065-y

Zhao, J., \& Gallant, D. J. (2012). Student evaluation of instruction in higher education: exploring issues of validity and reliability. Assessment \& Evaluation in Higher Education, 37(2), 227-235. https://doi.org/10.1080/02602938.2010.523819 